\title{
Dynamics of non-equilibrium charge carriers in p-germanium doped by gallium
}

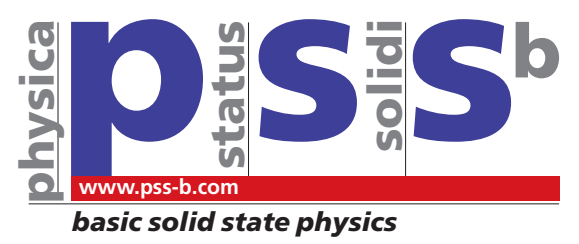

\author{
Nils Deßmann ${ }^{*, 1}$, S. G. Pavlov², V. V. Tsyplenkov' ${ }^{3}$ E. E. Orlova ${ }^{3}$, A. Pohl', V. N. Shastin ${ }^{3,4}$, \\ R. Kh. Zhukavin ${ }^{3}$, S. Winnerl ${ }^{5}$, M. Mittendorff ${ }^{5,6}$, J. M. Klopf ${ }^{5}$, N. V. Abrosimov7, \\ H. Schneider, and H.-W. Hübers ${ }^{1,2}$ \\ ${ }^{1}$ Department of Physics, Humboldt-Universität zu Berlin, Newtonstr. 15, 12489 Berlin, Germany \\ ${ }^{2}$ Institute of Optical Sensor Systems, German Aerospace Center (DLR), Berlin, Germany \\ ${ }^{3}$ Institute for Physics of Microstructures, Russian Academy of Sciences, Nizhny Novgorod, Russia \\ ${ }^{4}$ Nizhny Novgorod State University, Nizhny Novgorod, Russia \\ ${ }^{5}$ Helmholtz-Zentrum Dresden-Rossendorf, Dresden, Germany \\ ${ }^{6}$ Institute for Research in Electronics and Applied Physics, University of Maryland, Maryland, USA \\ ${ }^{7}$ Leibniz-Institute for Crystal Growth, Berlin, Germany
}

Received 25 November 2016, revised 16 January 2017, accepted 17 January 2017

Published online 20 February 2017

Keywords acceptor states, electronic capture, free electron laser, germanium, pump-probe experiment

*Corresponding author: e-mail nils.dessmann@dlr.de, Phone: +49 3067055 7921, Fax: +49 306705550

The low-temperature capture processes of non-equilibrium holes into gallium acceptors in moderately doped p-germanium $\left(N_{\mathrm{A}} \approx 2 \times 10^{15} \mathrm{~cm}^{-3}\right)$ has been investigated by a single-color pump-probe experiment using the free electron laser FELBE. The capture time decreases with increasing average photon flux density of the excitation pulse from about $10.9 \mathrm{~ns}$ ( at $\sim 1.2 \times 10^{24} \mathrm{~cm}^{-2} \mathrm{~s}^{-1}$ ) to $\sim 1.2 \mathrm{~ns}\left(\sim 2 \times 10^{26} \mathrm{~cm}^{-2} \mathrm{~s}^{-1}\right)$. Relaxation inside the valence band is almost independent on pump light intensity and its characteristic time is about 200 ps. In Addition, the intracenter relaxation times of the lowest excited Ga states were measured. The lifetimes scale with the phonon density of states controlling the bound hole - acoustic phonon interaction. The lifetime of the lowest excited state, $1 \Gamma_{8}^{-}$, was measured to be $\sim 275 \mathrm{ps}$; while the lifetimes of the higher excited states, $2 \Gamma_{8}^{-}$and $3 \Gamma_{8}^{-}$, were found to be $\sim 160 \mathrm{ps}$.
1 Introduction Germanium $(\mathrm{Ge})$ doped with gallium (Ge:Ga) is a widely used semiconductor for sensitive lowtemperature photon detectors of terahertz $(\mathrm{THz})$ radiation at frequencies from approximately 1.5 to $6 \mathrm{THz}(200-50 \mu \mathrm{m})$ $[1,2]$. The high carrier mobility, low lattice absorption at $\mathrm{THz}$ frequencies, and the possibility to precisely control of the doping make Ge a unique material for broadband and sensitive photodetectors. The most sensitive detection mechanism is based on photoconductivity induced by free charge carriers, which are generated by absorption of $\mathrm{THz}$ radiation on transitions from the Ga ground state $\left(1 \Gamma_{8}^{+}\right)$into the valence band (photo-ionization) and into excited $\mathrm{Ga}$ states (photoelectric excitation) (Fig. 1). The longwavelength cutoff of these detectors is determined by the energy gap between the impurity ground state and the high odd-parity excited states in the vicinity of the valence band (VB) of $\sim 10 \mathrm{meV}$. For Ge:Ga this corresponds to $\sim 120 \mu \mathrm{m}[1,2]$. It can be extended to $\sim 200 \mu \mathrm{m}$ by applying an uniaxial compressive force [3]. Spectrometers and photometers, which rely on these detectors, have been used in astronomy in a number of space- and airborne observatories. The most recent example is FIFI-LS, the Field-Imaging Far-Infrared Line Spectrometer. This spectrometer is equipped with two $16 \times 25$ pixel Ge:Ga detector arrays. One covers the range from 50 to $125 \mu \mathrm{m}$ and the other one the range from 105 to $200 \mu \mathrm{m}$. Since 2014 it is in operation on SOFIA, the Stratospheric Observatory for Infrared Astronomy [4, 5].

Despite the wide use of this type of detector there is relatively little experimental evidence on the relaxation of photo-excited carriers into attractive Coulomb centers in nand p-type Ge. While in n-Ge crystals interaction with intervalley phonons can contribute to the large relaxation rates, in $\mathrm{p}-\mathrm{Ge}$ crystals direct relaxation from degenerated 
FEL Power (normalized)

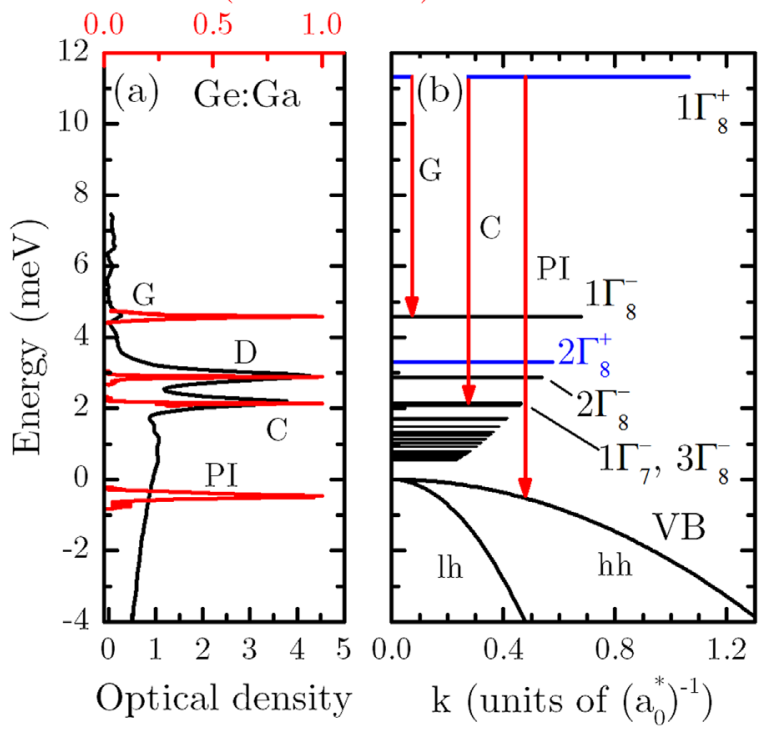

Figure 1 (a) Absorption spectrum of the investigated Ge:Ga sample (black line) and normalized FEL output spectra at the photon energies of the investigated transitions (red lines). The energy scale is set to zero at the $\Gamma$-point of the valence band. The spectral features correspond to intracenter transitions (indicated by G, D, and C) [9]. (b) Energy diagram for Ge:Ga with FEL excitation transitions, wavenumber $k$ is in units inversed to the effective Bohr radius $a_{0}{ }^{*}$. Discrete energy levels of Ga acceptors in Ge lie in the Ge bandgap; electronic states in vicinity of the minimum of the valence band are degenerated in the light $(l h)$ and heavy hole $(h h)$ subbands [9]. The red arrows down indicate photoionization (PI) and photo-excitation of bound holes by FEL radiation at wavelengths of $105 \mu \mathrm{m}$ (PI), $135 \mu \mathrm{m}$ (C), $150 \mu \mathrm{m}$ (D), and $184 \mu \mathrm{m}(\mathrm{G})$.

light $(l h)$ and heavy hole $(h h)$ subbands with emission of an optical phonon can be expected. A detailed understanding of these processes is important, as they ultimately limit sensitivity and speed of such detectors.

For clarity, we shall denote the relaxation of a free hole from the valence band bottom into the ground impurity state as a capture process, the decay of a photo excited free hole within the valence band as intraband relaxation, and the relaxation of the bound charge carrier from excited into the ground $\mathrm{Ga}$ state as intracenter relaxation.

The low-temperature $(T=5-40 \mathrm{~K})$ capture of free electrons in moderately doped $\mathrm{n}-\mathrm{Ge}$ ( $\mathrm{Sb}$ concentration $\sim 1 \times 10^{15} \mathrm{~cm}^{-3}$ ) has been studied by a time-resolved pump-probe experiment at $\sim 3 \mathrm{THz}$ [6]. It revealed a typical capture time of approximately $1.7 \mathrm{~ns}$. An additional intraband relaxation process with a characteristic time of approximately 200 ps occurs if electrons are excited above the bottom of the conduction band. This excitation can be achieved either by two-photon optical excitation, or, alternatively, by depopulation of the ground state due to elevated lattice temperature or electric impurity breakdown. Furthermore, the relaxation times of photo-excited free charge carriers in heavily doped ( $\mathrm{Sb}$ and $\mathrm{Ga}$ concentration $\left.(2-5) \times 10^{16} \mathrm{~cm}^{-3}\right)$ and highly compensated Ge was studied [7]. The dominant dopant was either $\mathrm{Ga}(\mathrm{p}-\mathrm{Ge}: \mathrm{Ga}: \mathrm{Sb})$ or $\mathrm{Sb}$ (n-Ge:Sb:Ga) with compensating doping levels close to $100 \%$. Compensation results in a larger number of attracting centers for photo-excited electrons, which enhances the capture process. The relaxation time is a function of pump pulse fluence and compensation and ranges from 30 to 300 ps. A fast photoconductive detector made from this material shows a response time of $150 \mathrm{ps}$ on the order of its fundamental relaxation time [7].

In this paper, we present the time-resolved investigation of different relaxation mechanisms of photoexcited and photoionized carriers in $\mathrm{p}$-Ge crystals moderately doped with $\mathrm{Ga}\left(\sim 2 \times 10^{15} \mathrm{~cm}^{-3}\right)$ with a low compensation (below $1 \%)$. The measurements were performed with a dedicated pump-probe setup [8] at the free electron laser (FEL) FELBE of the Helmholtz-Zentrum Dresden-Rossendorf. In addition, the lifetimes of three bound excited states were measured by this method.

2 Sample characterization and measurement details The p-Ge:Ga crystal was Czochralski-grown and doped from the melt. A p-Ge:Ga sample with dimensions of $10 \times 10 \times 1 \mathrm{~mm}^{3}$ cut from this crystal was used for the measurement. It was wedged to an angle of $\sim 0.5^{\circ}$ in order to avoid light interference in the sample. Low-temperature absorption spectra were recorded with a Bruker Fourier transfrom infrared spectrometer (FTIR) Vertex $80 \mathrm{~V}$. For this purpose the sample was placed in a Janis ST-100-FTIR liquid helium (He) flow-cryostat and the temperature was measured with a Lakeshore Model 331 temperature controller by a sensor mounted on the cold finger in vicinity to the sample. The temperature was varied between 5 and $60 \mathrm{~K}$ with a resistive heater attached to the sample holder. The strongest impurity transitions observed in the absorption spectra are between the ground state $\left(1 \Gamma_{8}^{+}\right)$and the lower excited states $\left(1 \Gamma_{8}^{-}, 2 \Gamma_{8}^{-}\right.$, and $\left.3 \Gamma_{8}^{-}\right)$, labeled in accordance with Ref. [9] as G, D, and C, respectively (Figs. 1 and 2). This confirms Ga centers to be the dominant dopant species. In Fig. 1 we choose the common energy zero position at the maximum (top) of the VB. Then, the bound Ga states, lying in the energy bandgap, have positive values of binding energies with the $\mathrm{Ga}$ ground state having an ionization energy of $11.32 \mathrm{meV}$. The optical density (Fig. 1) is a product $\sigma d N$, where $\sigma$ is the absorption cross section, $d$ is the sample's thickness, and $N$ is the number density of absorption centers. The optical densities range from almost zero below photon energies corresponding to the G-line to about 4.5 at the maximum of the D-line (Fig. 1).

To investigate the absorption processes in the Ge:Ga sample, we measured the temperature dependent absorption spectra between 5 and $60 \mathrm{~K}$ in temperature steps of $\sim 2.5 \mathrm{~K}$ (Fig. 2). The occurrence of more than one absorption process leads to the superposition of their optical densities, $\sum_{j} \sigma_{j} d N_{j}$. Assuming constant $\sigma_{\mathrm{j}}$ 's at a certain FEL wavelength $\lambda_{\mathrm{FEL}}$, each absorption process can be seen as a measure of the number of the respective absorption centers 


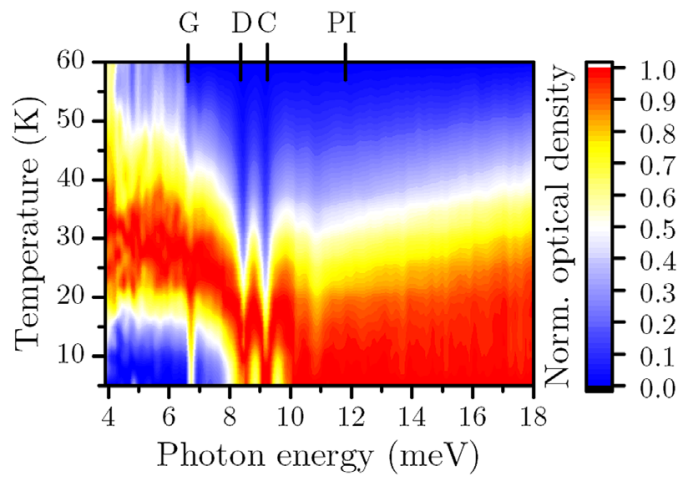

Figure 2 Absorption spectra of the investigated Ge:Ga sample at different temperatures from 5 to $60 \mathrm{~K}$. The spectra are normalized on maximal optical density for each temperature.

$N_{\mathrm{j}}$. For further comparison the optical density was normalized for each temperature.

At photon energies below the G-line $(<6 \mathrm{meV})$ the optical density peaks at about $30 \mathrm{~K}$ and disappears for temperatures above $50 \mathrm{~K}$. This indicates the existence of $(l h-h h)$ inter-valence-band absorption and absorption on transitions between high excited Ga states, which arise from thermal excitation of the Ga centers. At higher photon energies this maximum shifts to lower temperatures and peaks when the FEL wavelength is in resonance with one of the intracenter lines.

For measuring the capture time when the carrier is excited into the valence band the $\lambda_{\text {FEL }}$ was $105 \mu \mathrm{m}$ (Fig. 1a). This corresponds to a photon energy $(11.79 \mathrm{meV})$, larger than the ionization energy of the Ga centers, and it fits to an atmospheric window. For intracenter excitation the experiment was repeated with the $\lambda_{\mathrm{FEL}}$ that match the G$\left(1 \Gamma_{8}^{+} \rightarrow 1 \Gamma_{8}^{-}\right)$, D- $\left(1 \Gamma_{8}^{+} \rightarrow 2 \Gamma_{8}^{-}\right)$, and the C-line $\left(1 \Gamma_{8}^{+} \rightarrow\right.$ $\left.3 \Gamma_{8}^{-}\right)$at $184 \mu \mathrm{m}(6.74 \mathrm{meV}), 150 \mu \mathrm{m}(8.27 \mathrm{meV})$, and $135 \mu \mathrm{m}(9.18 \mathrm{meV})$, respectively. The pump-probe experiment is based on the time-resolved measurement of photoinduced transmission change, $\Delta T$, of the sample. In the case of Ge:Ga this is caused by a non-equilibrium carrier population. The change in transmission as a function of the time delay between exciting (pump) and analyzing (probe) beams is a measure of the dynamics of the system toward equilibrium. In the experiment the incoming FEL radiation is split into a strong pump pulse and a weaker probe pulse using a Mylar beam splitter. The time delay of the probe pulse with respect to the pump pulse was realized with an optical delay stage in the probe beam path. FELBE provides pulses at a repetition rate of $13 \mathrm{MHz}$ in a quasi-continuous mode and, hence, allows for the use of lock-in detection. The pump-probe signal (PPS), that is the pump-induced change of the probe beam transmitted through the sample, is measured by modulating the pump beam and recording the signal of the probe beam with a silicon bolometer. The mechanical chopping frequency in the pump beam path was set to $\sim 360 \mathrm{~Hz}$. Due to absorption by water vapor in the probe beam path the signal has a systematic drift caused by the variation of the optical path length during the optical delay stage drive. This effect is compensated using a reference signal obtained by blocking the pump beam in front of the sample and measuring the lock-in signal of the modulated probe beam as a function of delay stage position. The relative change in transmission of the sample induced by the pump, $\Delta T / T$, is obtained by dividing the PPS and the reference signal. This will be referred to as "relative PPS" in the text. For the measurements, the sample was placed in a liquid He flow-cryostat and cooled to about $5 \mathrm{~K}$, in order to ensure that the holes are bound to the ground state of the $\mathrm{Ga}$ centers. The cryostat was equipped with wedged diamond windows which have a flat transmission over the whole frequency range necessary for our measurements.

At the wavelengths of our experiments $(105,135,150$, and $184 \mu \mathrm{m}$ ) the pulses are quasi-Gaussian shaped with a full width at half-maximum (FWHM) ranging from $\sim 10 \mathrm{ps}$ at $105 \mu \mathrm{m}$ to $\sim 18$ ps at $184 \mu \mathrm{m}$. Pump and probe beams were focused onto the sample using a $10 \mathrm{~cm}$ focal-length off-axis parabolic mirror down to a spot of $\sim 400 \mu \mathrm{m}$ in diameter. They are slightly tilted $\left(\sim 10^{\circ}\right)$ with respect to each other. The average FEL power was measured with a pyroelectric powermeter (Ophyr). Using the average power, spot size, pulse duration, reflection losses at the vacuum window and sample surfaces the average photon flux density of the pump pulse, $\Phi_{\text {pump }}$, was estimated. $\Phi_{\text {pump }}$ was varied over a large range from $1.2 \times 10^{24}$ to $2 \times 10^{26} \mathrm{~cm}^{-2} \mathrm{~s}^{-1}$. The maximum average pump power available from the FEL in this experiment was $0.54 \mathrm{~W}$, which corresponds to $\Phi_{\text {pump }}$ $\sim 2 \times 10^{26} \mathrm{~cm}^{-2} \mathrm{~s}^{-1}$. It could be reduced with two sets of the diffractive attenuators in steps of 3,5 , and $10 \mathrm{~dB}$. The probe power was kept constant at $\sim 1.2 \times 10^{24} \mathrm{~cm}^{-2} \mathrm{~s}^{-1}$ (average power $\sim 2 \mathrm{~mW}$ ).

3 Capture of photo-ionized carriers Previous investigations on $\mathrm{n}-\mathrm{Ge}: \mathrm{Sb}$ revealed a considerable contribution of intraband absorption in a pump probe experiment of up to $20 \%$ due to a high population of the conduction band with non-equilibrium carriers at large $\Phi_{\text {pump. }}$. This ultimately leads to a bi-exponential temporal dependence of the measured PPS, especially at large $\Phi_{\text {pump }}[6]$. Intervalence-band transitions from the $l h$-subband to the $h h$-subband become considerably strong, if the light intensity is larger than $\sim 1 \mathrm{MW} \mathrm{cm}^{-2}$ [10]. According to Ref. [11] the absorption coefficient for transitions from the $h h$ to the $l h$ subband, $\alpha_{\mathrm{hl}}$, can be estimated as follows:

$$
\alpha_{\mathrm{hl}}=8.25 T_{1} \sqrt{\lambda_{\mathrm{FEL}}} e^{(\mu-0.125) / k_{B} T_{1}} .
$$

Here, $T_{1}$ is the lattice temperature in $[\mathrm{K}], k_{\mathrm{B}}$ is the Boltzmann constant, $\lambda_{\mathrm{FEL}}$ in $[\mu \mathrm{m}]$, and $\mu$ is the chemical potential in [meV]. The equilibrium absorption cross section can then be obtained by dividing $\alpha_{\mathrm{hl}}$ by the equilibrium hole concentration in the $h h$ subband [11]. For typical values $\left(T_{1}=5 \mathrm{~K}, N_{\mathrm{A}}=2 \times 10^{15} \mathrm{~cm}^{-3}\right.$, $\left.\lambda_{\mathrm{FEL}}=105 \mu \mathrm{m}\right)$ the $\alpha_{\mathrm{hl}}$ is about one order of magnitude larger than the indirect intra-conduction-band absorption cross section in the case of $\mathrm{n}-\mathrm{Ge}: \mathrm{Sb}$. It contributes about $50 \%$ to the 
total absorption cross section of p-Ge:Ga. A strong $\Phi_{\text {pump }}$ causes a large number of ionized Ga centers, $N_{\mathrm{i}}$. By twophoton optical excitation, a fraction of the non-equilibrium carriers at the VB edge will be further excited by intervalence-band absorption to the $l h$-subband. The scattering of hot holes, $N_{\mathrm{h}}$, from the $l h$-subband to the $h h$-subband is given by $[12,13]$ :

$$
\tau_{\mathrm{lh}}^{i}=-\frac{2^{3 / 2} m_{1} \varepsilon^{2}}{3 \pi e^{4} N_{\mathrm{i}}} \frac{|E|^{3 / 2}}{m_{\mathrm{h}}^{1 / 2}}\left(2+\phi \ln \frac{\phi-1}{\phi+1}\right)^{-1},
$$

with $\phi=\frac{1+m_{\mathrm{h}} / m_{1}}{2 \sqrt{m_{\mathrm{h}} m_{1}}}, m_{\mathrm{h}}$ and $m_{1}$ are the effective mass of holes in the $h h$ - and in the $l h$-subband, correspondingly, $\varepsilon$ is the dielectric permittivity, and $|E|$ is the hole's energy with respect to the bottom of the valence band (here $|E| \sim 12 \mathrm{meV}$ for $\left.\lambda_{\text {FEL }}=105 \mu \mathrm{m}\right)$. The scattering rate of this process is estimated to be about $2 \times 10^{11} \mathrm{~s}^{-1}$ and dominates the relaxation in the first few ps after excitation with a FEL pulse. The majority of hot holes will, therefore, relax to the $h h$-subband by the emission of acoustic phonons. This process can be estimated by an approach which is described in Ref. [14]. For a hole energy I $E \mathrm{l}=12 \mathrm{meV}$, which is approximately the ionization energy of the impurity centers, a $h$-intraband relaxation rate of $(4-8) \times 10^{9} \mathrm{~s}^{-1}$ is obtained at temperatures from 5 to $10 \mathrm{~K}$. The latter corresponds to the intraband relaxation time $\tau_{\mathrm{h}} \sim 125-250 \mathrm{ps}$.

We have measured the relative PPS at $\lambda_{\mathrm{FEL}}=105 \mu \mathrm{m}$ (11.8 meV) as a function of $\Phi_{\text {pump. }}$ In Fig. 3 three relative PPS of the Ge:Ga sample are shown for low $\left(1.2 \times 10^{24} \mathrm{~cm}^{-2} \mathrm{~s}^{-1}\right)$, medium $\left(2.2 \times 10^{25} \mathrm{~cm}^{-2} \mathrm{~s}^{-1}\right)$, and high $\left(2 \times 10^{26} \mathrm{~cm}^{-2} \mathrm{~s}^{-1}\right)$ $\Phi_{\text {pump. }}$ In order to obtain the characteristic time constants of both effects, hole capture and intraband relaxation, we fit the measured relative PPS, $\Delta T / T$, with a function $S(t)$, which is a convolution of the Gaussian-shaped probe pulse exp $\left(-\left(t-t_{0}\right)^{2} /\left(2 \Delta t^{2}\right)\right)$, here $\Delta t$ is the Gaussian RMS width of the probe pulse and $t_{0}$ is the time of maximum overlap between pump and probe pulses, with the probe transmission through the sample. The transmission is determined by the population of the valence band bottom, $N_{\mathrm{i}}(t) \sim \exp \left(-t / \tau_{\mathrm{c}}\right)$, and the hot hole concentration $N_{\mathrm{h}}(t) \sim \exp \left(-t / \tau_{\mathrm{h}}\right)$. Here $\tau_{\mathrm{c}}=\tau_{\mathrm{c}}\left(N_{\mathrm{i}}^{0}\right)$ is the capture time, $N_{\mathrm{i}}^{0}=N_{\mathrm{i}}(0)$, and $\tau_{\mathrm{h}}$ is the intraband relaxation time of hot holes down to the bottom of the valence band. Furthermore, a third component in the relative PPS with a characteristic time $\tau_{\mathrm{a}}$, sensitive to the alignment of the setup and primarily visible at large $\Phi_{\text {pump }}$, had to be taken in to account. This effect is known in the literature as the "coherent artifact". It can be caused for instance by pump-induced phase gratings in the sample $[15,16]$. The fit equation is then given by

$$
S(t)=\mathrm{e}\left[-\frac{\left(t-t_{0}\right)^{2}}{2 \Delta t^{2}}\right] *\left(a \cdot e^{-\frac{t}{t_{\mathrm{c}}}}+b \cdot e^{-\frac{t}{t_{\mathrm{h}}}}+c \cdot e^{-\frac{t}{t_{\mathrm{a}}}}\right) .
$$

Here, $\Delta t, t_{0}, a, b, c, \tau_{\mathrm{c}}, \tau_{\mathrm{i}}, \tau_{\mathrm{h}}$ are fit parameters and ${ }^{*}$ denotes a convolution.
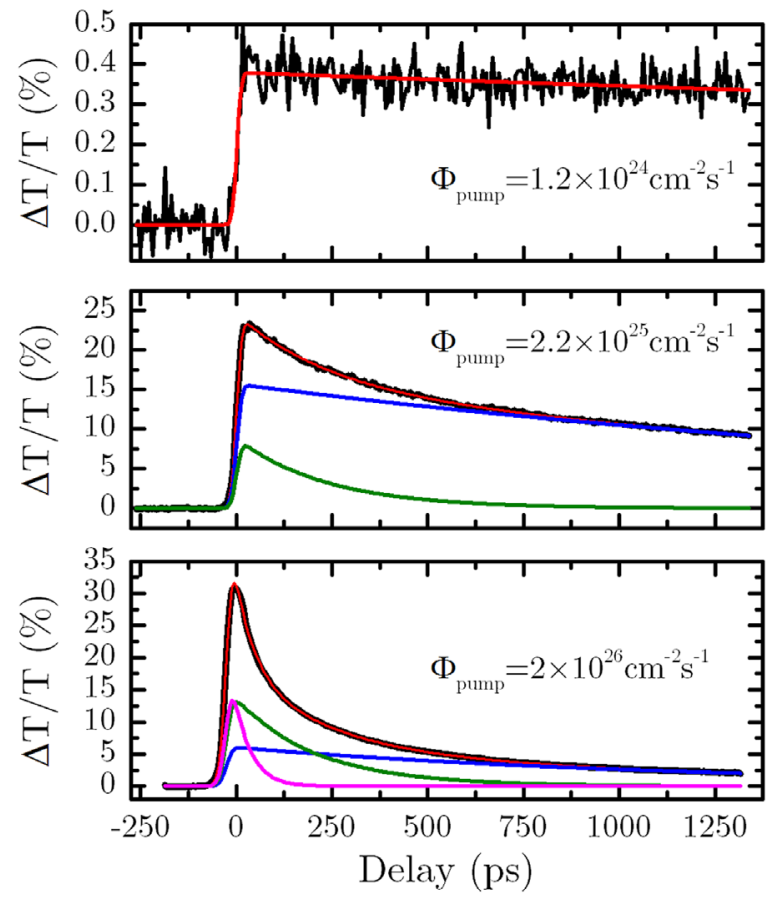

Figure 3 Pump-probe signals (black lines) of the Ge:Ga sample obtained at $\lambda_{\mathrm{FEL}}=105 \mu \mathrm{m}, \Phi_{\text {pump }}$ of $1.2 \times 10^{24}, 2.2 \times 10^{25}$, and $2 \times 10^{26} \mathrm{~cm}^{-2} \mathrm{~s}^{-1}$. The red lines are fits with Eq. (3). The temperature of the sample was $\sim 5^{\circ} \mathrm{K}$. The blue, magenta, and green lines are mono-exponential contributions to the relative PPS obtained from the fit. They show two processes, which are ascribed to intraband relaxation (green line) and hole capture by ionized centers (blue line). At low pump intensity, a mono-exponential fit (red = blue) is sufficient. At large $\Phi_{\text {pump }}$ (lowest graph) the signal is fitted with three components, the third component (magenta) is ascribed to the coherent artifact.

At low $\Phi_{\text {pump }}=1.2 \times 10^{24} \mathrm{~cm}^{-2} \mathrm{~s}^{-1}$ (top graph in Fig. 3 ) the signal can be fitted with a mono-exponential function with $\tau_{\mathrm{c}}=11 \mathrm{~ns}$. This process is the capture of holes by ionized centers. As $\Phi_{\text {pump }}$ is increased to $2.2 \times 10^{25} \mathrm{~cm}^{-2}$ $\mathrm{s}^{-1}$ a two-exponential fit is required (middle graph in Fig. 3). The main contribution (about $66 \%$ of the total PPS) is the hole capture but with a much shorter characteristic time: $\tau_{\mathrm{c}} \sim 3 \mathrm{~ns}$. The second contribution of the PPS contributes about $33 \%$ to the total signal. The characteristic time $\tau_{\mathrm{h}}$ is $\sim 200$ ps. This can be assigned to intraband relaxation. By going to higher $\Phi_{\text {pump }}=2 \times 10^{26} \mathrm{~cm}^{-2} \mathrm{~s}^{-1}$ (bottom graph in Fig. 3) $\tau_{\text {c }}$ drops to $\sim 1 \mathrm{~ns}$. At this high photon flux the photoinduced occupation of the valence band is so large, that the intraband relaxation dominates with about $66 \%$ of the total PPS (excluding the contribution of the coherent artifact to the total signal). The characteristic time of the intraband relaxation $\tau_{\mathrm{h}}$ is within the measurement uncertainty independent of $\Phi_{\text {pump }}$ and has a value of about 200 ps.

The main recombination mechanism in moderately doped and low compensated Ge at low temperatures is the cascade capture via emission of acoustic phonons. The characteristic energy of an acoustic phonon is much smaller than the binding energy, thus excited carriers relax gradually 
through the ladder of excited impurity states. The model of cascade capture was originally developed for isolated Coulomb centers [17]. The centers can be regarded as isolated when their concentration is relatively low and the orbitals, which are significant for the capture process, do not overlap. In this case the capture time is inversely proportional to the concentration of impurity ions. This in turn is proportional to $\Phi_{\text {pump }}$, provided $\Phi_{\text {pump }}$ is low. The dependence of the capture time on $\Phi_{\text {pump }}$ is shown in Fig. 4. Up to $\Phi_{\text {pump }} \approx 4 \times 10^{25} \mathrm{~cm}^{-2} \mathrm{~s}^{-1}$ it is inversely proportional to the pump rate while above that value the dependence of the capture time on the pump rate is less pronounced. According to the cascade capture theory the capture time is proportional to $N_{\mathrm{i}}^{-1 / 6}$ for densely spaced ionized centers, that is if the orbits relevant for the capture process overlap. This explains qualitatively the dependence of capture times at high pump rates.

Figure 5 shows the peak value of the total PPS as well as the peak values of its two components for different $\Phi_{\text {pump }}$. The change of transmission of the maximum of the total PPS, increases with $\Phi_{\text {pump }}$ from 0.4 to $43 \%$. At large $\Phi_{\text {pump }}$ the modulation starts to saturate and peaks at $6.2 \times 10^{25} \mathrm{~cm}^{-2} \mathrm{~s}^{-1}$ with $43 \%$. Above $\Phi_{\text {pump }}=6.2 \times 10^{26} \mathrm{~cm}^{-2} \mathrm{~s}^{-1}$ the modulation drops rapidly apparently due to heating of the sample that decreases the Ga ground state population. The modulations related to impurity-to-band absorption, $\Delta T_{\max , 1} / T$, as well as to $(h h-l h)$ inter-valence-band absorption, $\Delta T_{\max , 2} / T$, show a similar dependence on the pump rate. However, the maximum of the modulation for $(h h-l h)$ inter-valence-band absorption is at higher $\Phi_{\text {pump }}$ while for impurity-to-band absorption it is at lower pump rate, because the first one requires a significant amount of holes in the valence band.

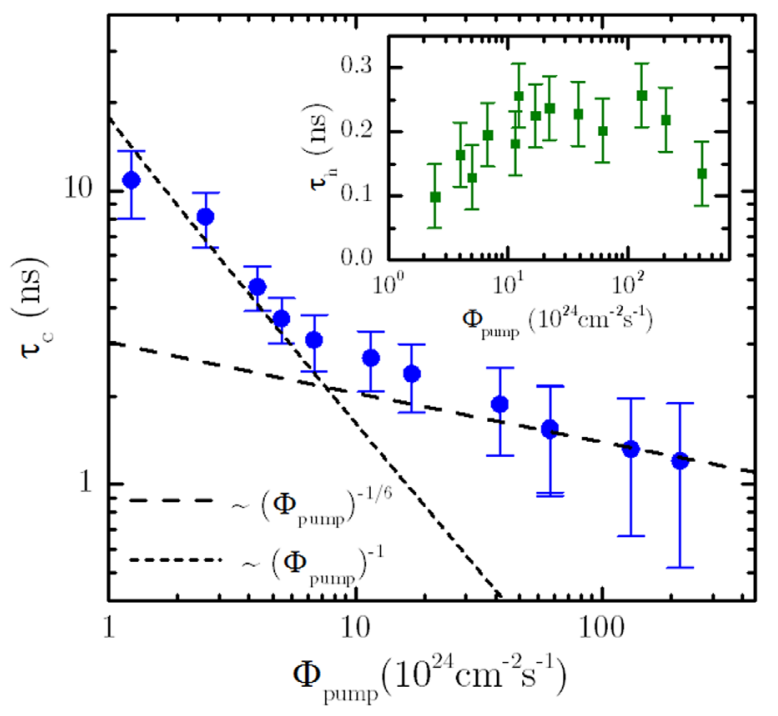

Figure 4 Capture time $\tau_{\mathrm{c}}$ as a function of the photon flux density $\Phi_{\text {pump }}, \lambda_{\text {FEL }}=105 \mu \mathrm{m}$ : experimental results and fits according to the cascade capture via emission of acoustic phonons with account of overlap of the orbits of capture centers. Inset: dependence of intraband relaxation time $\tau_{\mathrm{h}}$ on $\Phi_{\text {pump }}$.

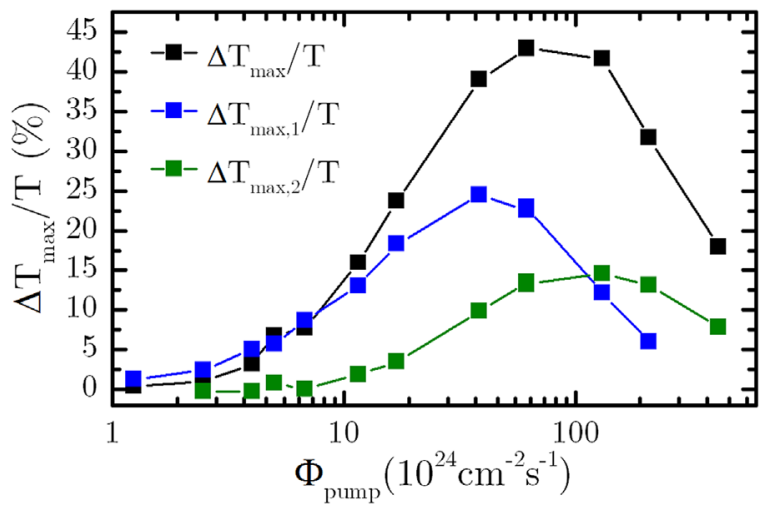

Figure 5 Dependence of the maximum of the relative transmission $\Delta T_{\text {max }} / T$ ) on the pump pulse energy at $\lambda_{\mathrm{FEL}}=105 \mu \mathrm{m}$. They show the contributions of two processes to the decay of the relative PPS, namely intraband relaxation $T_{2}$ (green line) and hole capture by ionized centers $T_{1}$ (blue line). $T=T_{1}+T_{2}$.

This leads to the dominance of the $(l h-h h)$ inter-valence-band absorption at $\Phi_{\text {pump }}$ larger than $\sim 1 \times 10^{26} \mathrm{~cm}^{-2} \mathrm{~s}^{-1}$.

We summarize a five step photoionization-recombination process for non-equilibrium carriers in p-Ge (Fig. 6) with the following derived relaxation rates: (i) Neutral Ga centers are ionized by the FEL radiation and holes are generated in the $h h$ valence subband. (ii) Subsequent excitation by $h h-l h$ absorption transitions occurs, clearly detectable when reaching a critical carrier concentration in the $h h$ valence subband. (iii) The scattering between $l h$ and $h h$ subband is very efficient (rate estimate $2 \times 10^{11} \mathrm{~s}^{-1}$ ) and leads to the population singularity in the $h h$ subband at about twice the photon excitation energy with respect to the impurity ground state. (iv) The relaxation down the $h h$ subband by emission of acoustical phonons occurs on a typical time scale of $\sim 200 \mathrm{ps}$ (fixed rate $5 \times 10^{9} \mathrm{~s}^{-1}$ ). (v)

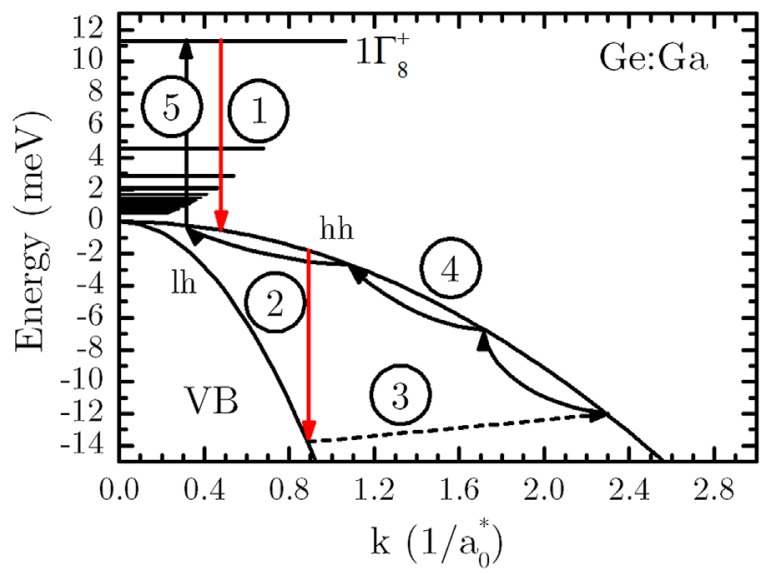

Figure 6 Five step photoionization-recombination processes as identified in the FEL pump-probe experiments for $\mathrm{p}-\mathrm{Ge}: \mathrm{Ga}$. The subsequent two-step excitation from the impurity ground state $1 \Gamma_{8}^{-}$ to the $l h$-subband $(1,2)$ is followed by $l h-h h$ scattering $(3)$ and intraband relaxation (4) with the final capture back to the ground state (5). 
The cascade capture into the Coulomb potentials of the ionized hydrogenic centers is the last step to the impurity ground state (rates are excitation power dependent, $>10^{8} \mathrm{~s}^{-1}$ ). It is important to note that only a fraction of the ionized holes will pass the complete process. Depending on the degree of ionization, a number of carriers will be directly captured by the ionized center (v) after being pumped into the $h h$ subband (i), omitting the steps (ii) to (iv).

4 Intracenter recombination We measured lifetimes of the $1 \Gamma_{8}^{-}, 2 \Gamma_{8}^{-}$, and $3 \Gamma_{8}^{-}$Ga states by setting the FEL to the G-line transition at $184 \mu \mathrm{m}$, the D-line transition at $150 \mu \mathrm{m}$, as well as to the C-line transition at $135 \mu \mathrm{m}$ (Fig. 1). The spectral width of the FEL radiation was smaller than those of the Ga absorption lines. A component with a short time constant was present in all signals at wavelengths of 184 and $135 \mu \mathrm{m}$. We assign this component to the coherent artifact that was seen also in the measurements of the intraband relaxation processes at large $\Phi_{\text {pump. }}$. It is known that the width of the coherent artifact is similar to that of the incident pulse autocorrelation [18]. The fitting of the relative PPS was done according to Eq. (3), however, the fitting parameter $\tau_{\mathrm{a}}$ in Eq. (3) was restricted to values below the pulse width to avoid any numerical interaction on the derived lifetimes.

At $\lambda_{\mathrm{FEL}}=184 \mu \mathrm{m} \Phi_{\text {pump }}$ was varied over a range from $6 \times 10^{24}$ to $5 \times 10^{25} \mathrm{~cm}^{-2} \mathrm{~s}^{-1}$. The relative PPS for $\Phi_{\text {pump }}$ $=5 \times 10^{25} \mathrm{~cm}^{-2} \mathrm{~s}^{-1}$ (Fig. 7a) shows the bi-exponential character of the signal. The longer time constant, assigned to the decay of the $1 \Gamma_{8}^{-}$state population, is independent on $\Phi_{\text {pump }}$ and has an arithmetic mean value of $(275 \pm 59)$ ps (Fig. 7b). The FWHM duration of the FEL pulse derived from the fit was $18 \mathrm{ps}$ in all PPS.

At $\lambda_{\mathrm{FEL}}=150 \mu \mathrm{m} \Phi_{\text {pump }}$ was set to $2.5 \times 10^{24}$ and $5 \times 10^{24} \mathrm{~cm}^{-2} \mathrm{~s}^{-1}$. In both cases only a mono-exponential signal was observed. A relative PPS for $\Phi_{\text {pump }}=5 \times 10^{25}$ $\mathrm{cm}^{-2} \mathrm{~s}^{-1}$ is shown in Fig. 8a. The FWHM of the FEL pulse derived from the fit was $\sim 18 \mathrm{ps}$ in all relative PPS. The time constant of the population decay of the $2 \Gamma_{8}^{-}$state is consistent at both $\Phi_{\text {pump }}$ and shorter than the lifetime of the $1 \Gamma_{8}^{-}$state with a mean value of $(157 \pm 41)$ ps (Fig. 8b).

At $\lambda_{\mathrm{FEL}}=135 \mu \mathrm{m} \Phi_{\text {pump }}$ was varied from $3.2 \times 10^{25}$ to $1 \times 10^{26} \mathrm{~cm}^{-2} \mathrm{~s}^{-1}$. A relative PPS for $\Phi_{\text {pump }}=3.3 \times 10^{25}$ $\mathrm{cm}^{-2} \mathrm{~s}^{-1}$ is shown in Fig. 9a. Similar to the previous measurements, we observed a bi-exponential relative PPS. Here, the FWHM of the FEL pulse derived from the fit was $\sim 13 \mathrm{ps}$ in all relative PPS. The longer time constant which is identified with the population decay of the $3 \Gamma_{8}^{-}$state is independent on $\Phi_{\text {pump }}$. The lifetime of the $3 \Gamma_{8}^{-}$state has a mean value of (162 \pm 37$)$ ps (Fig. 9b).

As mentioned above, the cascade model describes the capture of free carriers by ionized centers as a sequence of small steps in energy space until the probability of thermal reemission into the continuum is practically zero $[17,19]$. For $\mathrm{p}-\mathrm{Ge}: \mathrm{Cu}$ it has been confirmed experimentally that a considerable portion of the carriers is in bound excited
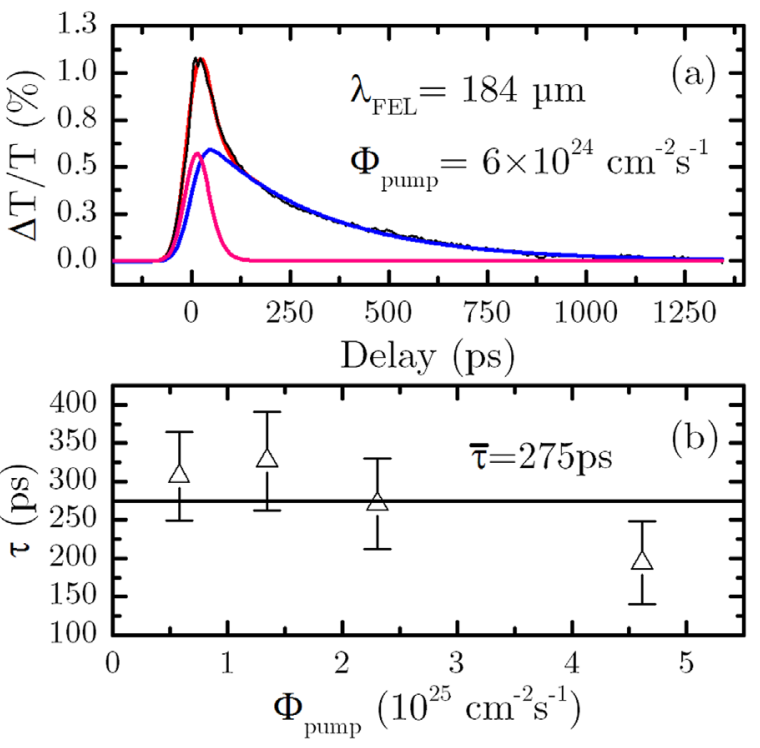

Figure 7 (a) Pump-probe signal (black line) of the Ge:Ga sample obtained at $\lambda_{\text {FEL }}=184 \mu \mathrm{m}$ and $\Phi_{\text {pump }}=4.6 \times 10^{25} \mathrm{~cm}^{-2} \mathrm{~s}^{-1}$. The red line is a fit with Eq. (3). The blue component of the biexponential fit is identified with the population decay of the $1 \Gamma_{8}^{-}$ state. The magenta line is identified with the coherent artifact. (b) Lifetime $\tau$ of the $1 \Gamma_{8}^{-}$state as a function of $\Phi_{\text {pump }}$ obtained from the fit of the relative PPS. The arithmetic mean value $\bar{\tau}$ is $274 \mathrm{ps}$.

acceptor states during the capture process [20]. The decrease of the probability of acoustic phonon assisted transitions with an increase of the transition energy is caused by the increase of the difference between the phonon momentum and the localization of impurity states in momentum space
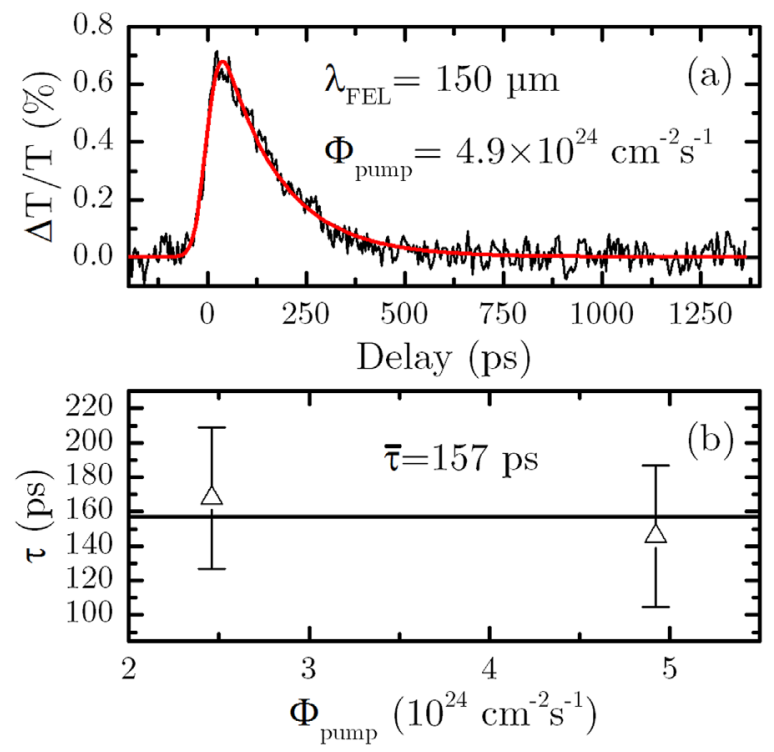

Figure 8 (a) Pump-probe signal (black line) of the Ge:Ga sample obtained at $\lambda_{\mathrm{FEL}}=150 \mu \mathrm{m}$ and $\Phi_{\text {pump }}=5 \times 10^{24} \mathrm{~cm}^{-2} \mathrm{~s}^{-1}$. The red line is a fit with Eq. (3). (b) Lifetime $\tau$ of the $2 \Gamma_{8}^{-}$state at two $\Phi_{\text {pump }}$ obtained from the fit of the pump probe signals. The arithmetic mean $\bar{\tau}$ is $157 \mathrm{ps}$. 

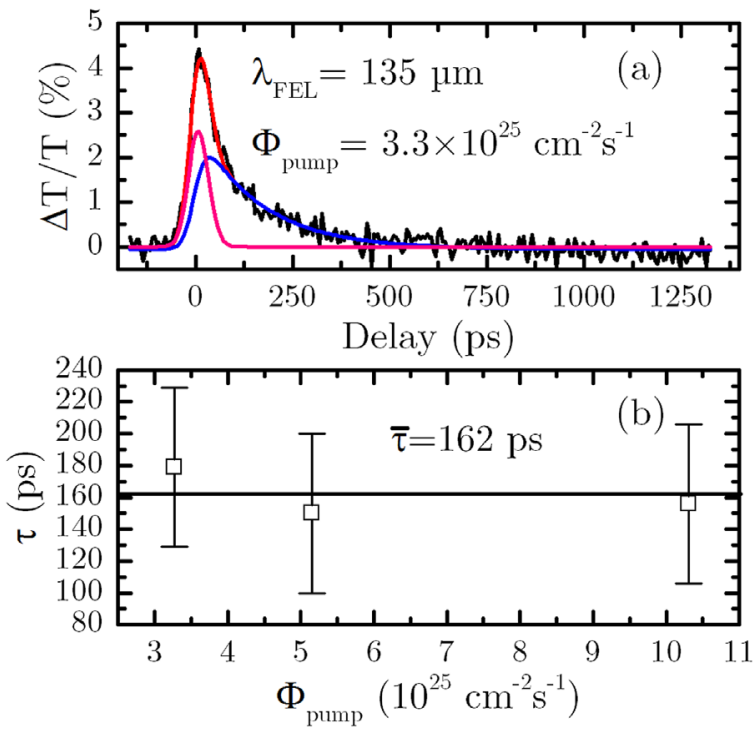

Figure 9 (a) Pump-probe signal (black line) of the Ge:Ga sample obtained at $\lambda_{\text {FEL }}=135 \mu \mathrm{m}$ and $\Phi_{\text {pump }}=3.3 \times 10^{25} \mathrm{~cm}^{-2} \mathrm{~s}^{-1}$. The red line is a fit with Eq. (3). The blue component of the biexponential fit is identified with the population decay of the $3 \Gamma_{8}^{-}$ state. The magenta line is identified with a coherent process. (b) Lifetime $\tau$ of the $2 \Gamma_{8}^{-}$state as a function of $\Phi_{\text {pump }}$ obtained from the fit of the pump probe signals. The arithmetic mean $\bar{\tau}$ is 162 ps.

[21]. The energy gaps between the first excited states are the largest, thus implying the largest lifetimes for these states. On the other hand, comprehensive studies of both shallow acceptors [22] and donors [23] in silicon by using a timeresolving pump-probe setup and a FEL, support the presence of direct transitions from the excited states to the ground state. A correlation between the one-phonon density of states (PDOS) of silicon and phonon assisted relaxation rates was noted in [22]. In Fig. 10 the PDOS [24]

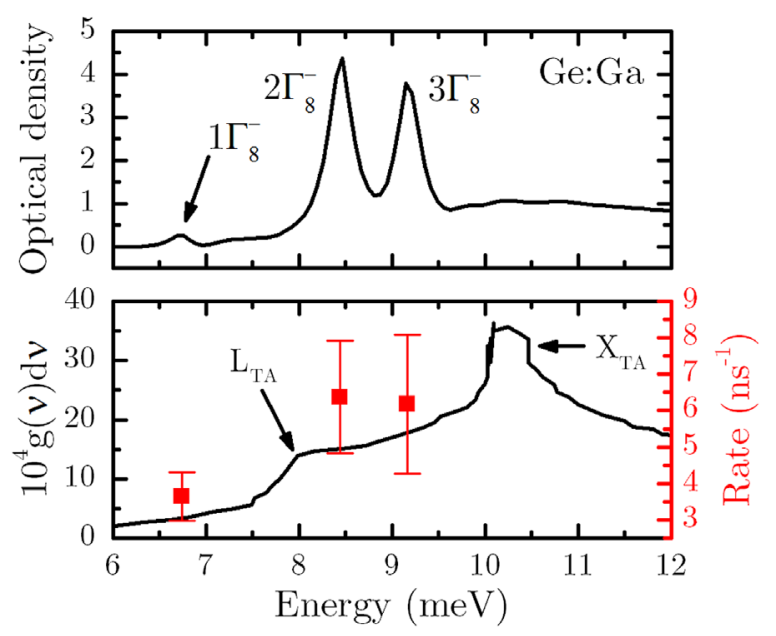

Figure 10 (a) Part of the absorption spectrum of the p-Ge:Ga sample, and (b) compared with the experimental relaxation rates and the PDOS, $\mathrm{g}(v) \mathrm{d} v(\mathrm{~d} v=0.01 \mathrm{THz})$ of Ge $(80 \mathrm{~K})$ [25]; arrows indicate energy of transversal acoustic (TA) phonons in Ge. of Ge is shown together with the absorption spectrum of the p-Ge:Ga sample. The G transition $\left(1 \Gamma_{8}^{-} \rightarrow 1 \Gamma_{8}^{+}\right)$is below the $\mathrm{L}_{\mathrm{TA}}$ phonon resonance whereas the $\mathrm{D}$ and $\mathrm{C}$ transitions $\left(2 \Gamma_{8}^{-}, 3 \Gamma_{8}^{-} \rightarrow 1 \Gamma_{8}^{+}\right)$are above that resonance; hence, the PDOS is larger. The $2 \Gamma_{8}^{-}$and $3 \Gamma_{8}^{-}$state are energetically above the $1 \Gamma_{8}^{-}$state and have an about $120 \mathrm{ps}$ shorter lifetime, while at about the same PDOS their lifetimes are equal. In accordance with the measurements for silicon, we attribute the difference of the lifetimes of excited states in p-Ge to the PDOS (Fig. 10) which at higher values leads to a more efficient phonon-assisted relaxation.

The lifetimes of $\mathrm{Ga}$ excited states which we have derived are order(s) of magnitude shorter than those reported for shallow acceptors in Ge in the experimental works. The calculated lifetime of the first excited state of an acceptor in Ge in Ref. [21] is about $100 \mathrm{~ns}$. Gershenzon et al. [25] have reported the lifetime of the $1 \Gamma_{8}^{-}$state in $\mathrm{p}-\mathrm{Ge}: \mathrm{B}$ to be as $\sim 60$ ns by analyzing line intensities of photoconductive spectra measured with a wide range of background illumination [26]. A theoretical analysis of acceptor quantum transitions supported this lifetime [25]. More accurate theoretical analysis of intracenter relaxation in $\mathrm{p}-\mathrm{Ge}$ is desirable.

5 Conclusions We have measured the lowtemperature capture of free holes into ionized $\mathrm{Ga}$ centers in moderately doped $\mathrm{p}-\mathrm{Ge}\left(N_{\mathrm{A}} \approx 2 \times 10^{15} \mathrm{~cm}^{-3}\right)$ using a single color pump-probe experiment and the free electron laser FELBE. The capture time decreases with increasing pump photon flux density from about $10.9 \mathrm{~ns}$ (at $\sim 1.2$ $\left.\times 10^{24} \mathrm{~cm}^{-2} \mathrm{~s}^{-1}\right)$ to $\sim 1.2 \mathrm{~ns}\left(\sim 2 \times 10^{26} \mathrm{~cm}^{-2} \mathrm{~s}^{-1}\right)$ due to an increase of the number of ionized impurity centers. We have shown that the capture rate is determined by the cascade model in the range of number of ionized impurity centers below $2 \times 10^{15} \mathrm{~cm}^{-3}$. With increasing pump power we observe a deviation from a sole cascade decay. The process with a characteristic time of $\sim 200 \mathrm{ps}$ is identified as an additional intraband relaxation process, caused by a subsequent excitation inside the valence band, similar to those previously observed for $\mathrm{n}-\mathrm{Ge}: \mathrm{Sb}$ [6]. Additionally, we have determined the lifetimes of the lowest $1 \Gamma_{8}^{-}$and the shallower $2 \Gamma_{8}^{-}$and $3 \Gamma_{8}^{-}$odd-parity excited states as 275 , 157 , and $162 \mathrm{ps}$, respectively. In contrast to [21, 26] we could not confirm the long lifetimes. Based on these results all excited states are likely to contribute in the recombination process. The $1 \Gamma_{8}^{-}$state has a binding energy below the $\mathrm{L}_{\mathrm{TA}}$ phonon resonance, that is in a region with a smaller PDOS compared to the $2 \Gamma_{8}^{-}$and $3 \Gamma_{8}^{-}$states, which lie above that resonance. Hence, our measurements suggest a direct dependence of the excited states lifetimes on the PDOS that determines a bound hole - acoustic phonon interaction.

Acknowledgements This research has been funded by the joint German-Russian program "Research on technological advances of radiation sources of photons and neutrons based on accelerators and neutron sources in cooperation with research organizations and universities of the Federal Republic of Germany" 
(InTerFEL project, BMBF No. 05K2014 and the Russian Ministry of Science and Education, unique identifier No. RFMEF161614 $\times 0008)$. N.D. and A.P. gratefully acknowledge support by the Helmholtz Research School on Security Technologies. We are grateful to P. Michel and the FELBE team for their dedicated support.

\section{References}

[1] E. E. Haller, Infrared Phys. Technol. 35, 127 (1994).

[2] E. Bründermann, H.-W. Hübers, and M. F. Kimmitt, Terahertz techniques, Springer Series in Optical Sciences (Springer, Berlin, Heidelberg, New York, 2012).

[3] A. G. Kazanskii, P. L. Richards, and E. E. Haller, Appl. Phys. Lett. 31, 496 (1977).

[4] S. Colditz, F. Fumi, N. Geis, R. Hönle, R. Klein, A. Krabbe, L. Looney, A. Poglitsch, W. Raab, M. Savage, F. Rebell, and C. Fischer, Proc. SPIE 8446, 844617 (2012).

[5] R. Klein, S. Beckmann, A. Bryant, S. Colditz, C. Fischer, F. Fumi, N. Geis, R. Hönle, A. Krabbe, L. Looney, A. Poglitsch, W. Raab, F. Rebell, and M. Savage, Proc. SPIE 9147, 91472X (2014).

[6] N. Dessmann, S. G. Pavlov, V. N. Shastin, R. K. Zhukavin, V. V. Tsyplenkov, S. Winnerl, M. Mittendorff, N. V. Abrosimov, H. Riemann, and H.-W. Hübers, Phys. Rev. B 89, 35205 (2014).

[7] N. Deßmann, S. G. Pavlov, A. Pohl, N. V. Abrosimov, S. Winnerl, M. Mittendorff, R. K. Zhukavin, V. V. Tsyplenkov, D. V. Shengurov, V. N. Shastin, and H.-W. Hübers, Appl. Phys. Lett. 106, 171109 (2015).

[8] S. Winnerl, M. Orlita, P. Plochocka, P. Kossacki, M. Potemski, T. Winzer, E. Malic, A. Knorr, M. Sprinkle, C. Berger, W. A. de Heer, H. Schneider, and M. Helm, Phys. Rev. Lett. 107, 237401 (2011).

[9] A. K. Ramdas and S. Rodriguez, Rep. Prog. Phys. 44, 1297 (1981).
[10] S. D. Ganichev, S. A. Emel'yanov, E. L. Ivchenko, E. Y. Perlin, Y. V. Terent'ev, A. V. Fedorov, and I. D. Yaroshetskii, Sov. Phys. JETP 64, 729 (1986).

[11] Y. T. Rebane, Sov. Phys. 14, 289 (1981).

[12] D. Kranzer, Phys. Status Solidi A 26, 11 (1974).

[13] M. I. D'yakonov and A. V. Khaetskii, Sov. Phys. JETP 59, 1072 (1984).

[14] M. Costato and L. Reggiani, Phys. Status Solidi B 58, 471 (1973).

[15] S. L. Palfrey and T. F. Heinz, J. Opt. Soc. Am. B 2, 674 (1985).

[16] Z. Vardeny and J. Tauc, Opt. Commun. 39, 396 (1981).

[17] V. N. Abakumov, V. I. Perel', and I. N. Yassievich, Sov. Phys. JETP 45, 354 (1977).

[18] H. Liu, H. Zhang, J.-H. Si, L.-H. Yan, F. Chen, and X. Hou, Chin. Phys. Lett. 28, 86602 (2011).

[19] M. Lax, Phys. Rev. 119, 1502 (1960).

[20] I. Wilke, O. D. Dubon, Jr, J. W. Beeman, and E. E. Haller, Solid State Commun. 93, 409 (1995).

[21] S. V. Meshov and I. Rashba, Sov. Phys. JETP 49, 1115 (1979).

[22] N. Q. Vinh, B. Redlich, A. F. G. van der Meer, C. R. Pidgeon, P. T. Greenland, S. A. Lynch, G. Aeppli, and B. N. Murdin, Phys. Rev. X 3, 11019 (2013).

[23] S. A. Lynch, P. T. Greenland, N. Q. Vinh, K. Litvinenko, B. Redlich, L. van der Meer, M. Warner, A. M. Stoneham, G. Aeppli, C. R. Pidgeon, and B. N. Murdin, in: 2008 5th IEEE Int. Conf. Group IV Photonics (IEEE, 2008), pp. 24-26.

[24] G. Nelin and G. Nilsson, Phys. Rev. B 5, 3151 (1972).

[25] E. M. Gershenzon, G. N. Gol'tsman, and N. G. Ptitsina, JETP Lett. 25, 539 (1977).

[26] E. M. Gershenzon, G. N. Gol'tsman, and N. G. Ptitsina, Sov. Phys. JETP 49, 711 (1979). 